\title{
أثر برنامج تدريبي في تمكين مفاهيم الثورة الصناعية الر ابعة لدى طلبة معهد العلوم الإسلامية بمسقط
}

\author{
لقمان بن خلفان المياحي'، نصربن ناصبر الجابري'، عبد الله بن علي الجههوري`، حسين بن علي الخروصيء \\ ' ماجستير طرق التدريس- مساعد مدير معهد العلوم الإسلامية بمسقط- سلطنة عُمان \\ r ماجستير طرق التدريس مدرس اللغة الإنجليزية بمعهد العلوم الإسلامية بمسقط - سلطنة عُمان

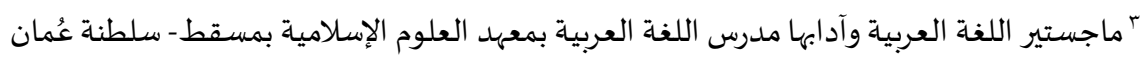

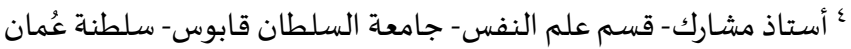 \\ 1 E-mail: luqman1233@gmail.com
}

هدفت الدراسة الحالية إلى التعرف على أثر برنامج تدريبي في تمكين مفاهيم الثورة الصناعية الرابعة لدى طلبة معهد العلوم الإسلامية بمسقط، وما إذا كانت هناك فروق دالة إحصائيا تعزى إلى متغيري الصف الدراسي والتوجه العلمي في تمكين مفاهيم الثورة الصناعية الرابعة، وتكونت

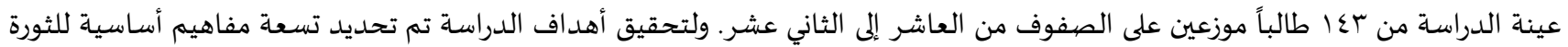

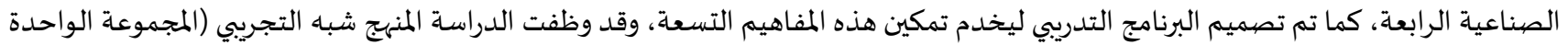

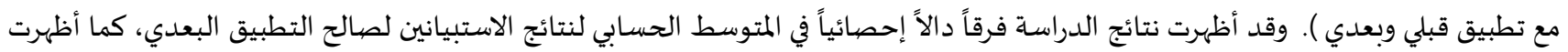
الدراسة فرقاً دالاً في أثر متغير الصف الديقي وفراسي. وخلصت الدراسة إلى مجموعة من المقترحات والتوصيات. الكلمات المفتاحية: الصناعية الرابعة؛ مفاهيم التمكين؛ برنامج التدريب.

\section{(ㄷ) (6)}

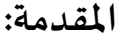

تُشكل الثورة الصناعية الرابعة قطب الرحى الذي تدور حولها الممارسات التنموية المعاصرة في الدول المتقدمة ودول العالم الثالث على حد

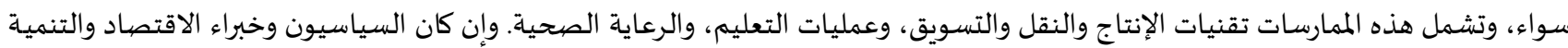
هم الذين يحملون لواء التغيير والتطوير إلا إنه يقع على عاتق القائمين على العملية التعليمية مسؤولية إعداد جيل قادية وادر على التعامل مع متغيرات هذه

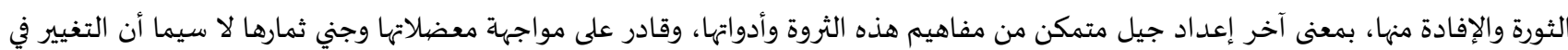

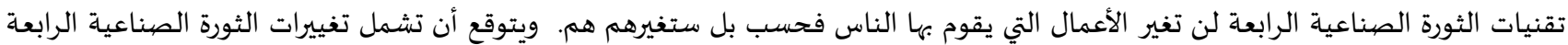

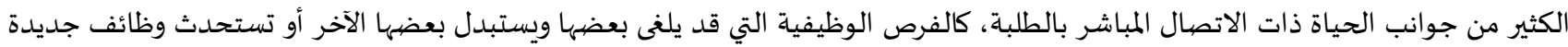

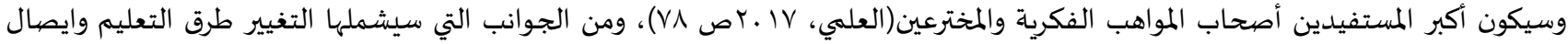

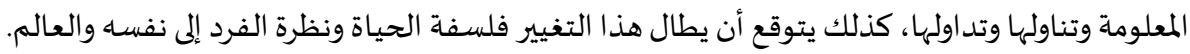

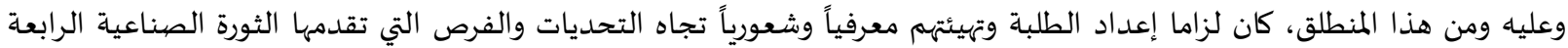

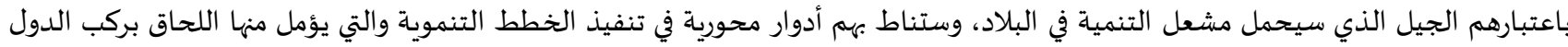

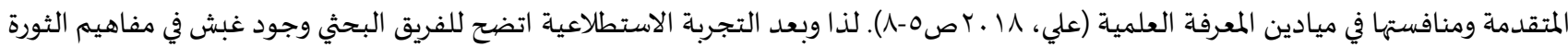
الصناعية لدى طلبة معهد العلوم الإسلامية بمسقط في سلطنة عمان، لذا تسعى الدراسة الحالية للتأكد من هذه الفرضية عبر الطرق العلمية المتاحة 
تتلخص مشكلة الدراسة في ملاحظة الفريق البحثي علامات لتدني تمكن طلبة معهد العلوم الإسلامية بمسقط في سلطنة عمان من مفاهيم الثورة الصناعية الرابعة وضعف في اطلاعهم على التحديات التي ستواجههم خلالها، كذلك لاحظ الفريق أيضاً- من خلال التجربة الاستطلاعية- عدم

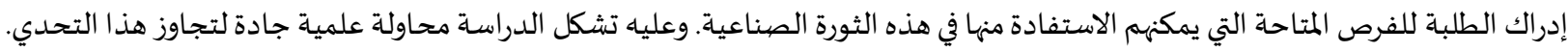

أسئلة الدراسـة: السؤال الأبرز في هذه الدراسة ما مدى فاعلية البرنامج التدريبي المقترح في تمكين مفاهيم الثورة الصيناعية الرابعة لدى طلبة معهد العلوم الإسلامية بمسقط؟ وينبثق من هذا السؤال الأسئلة الآتية:

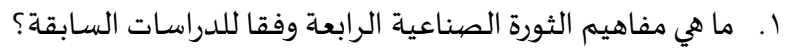

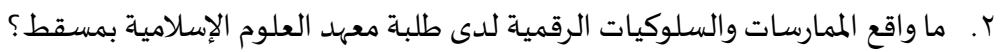

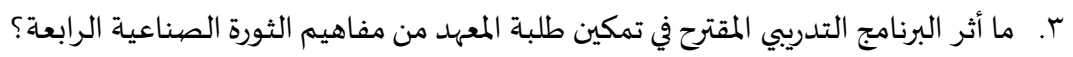

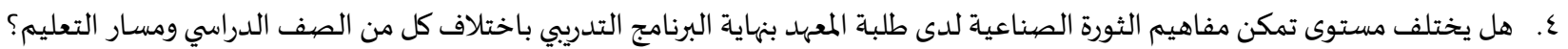

فرضيات الدراسـة:

ا. لا يوجد أثر للبرنامج التدريبي المقترح في تمكين مفاهيم الثورة الصناعية الرابعة لدى طلبة معهد العلوم الإسلامية بمسقط.

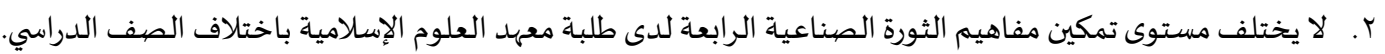
r. لا توجد فروق ذات دلالة إحصائية في مستوى تمكين مفاهيم الثورة الصناعية الرابعة لدى طلبة معهد العلوم الإسلامية بين المسار العلمي معائ والمسار الأدبي.

أهداف الدراسـة: تهدف الدراسة الحالية إلى:

ا. التعريف بالثورة الصناعية الرابعة وتغييراتها المتوقعة لدى طلبة معهد العلوم الإسلامية بمسقط. r. الإسهام في تهيئة الطلبة نفسيا ومعرفيا لمتطلبات الثورة الصنياعية.

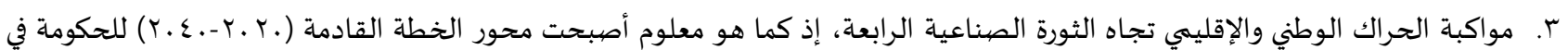
سلطنة عمان. ع. تقديم مفاهيم العمل والتعليم وفق منظور الثورة الصناعية الرابعة لدى الطلبة. 0. الإسهام في إيجاد حلول إجرائية لمشكلة عدم مواكبة الطلبة لمفاهيم الثورة الصناعية الرابعة.

أهمية الدراسـة:

ا. تمثل الدراسة بعدا نوعيا للدراسات الإجرائية بوصفها تسبر موضوعا على تماس مباشر بالخطط التنموية الوطنية، التي لا بد من مواكبتها،

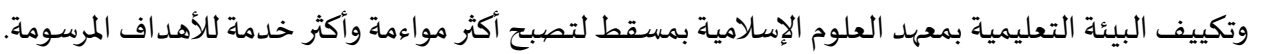
r. ت تنبع أهمية الدراسة من أهمية الموضوع المطروق وهو الثورة الصناعية الرابعة وتمكين مفاهيمها لدى الطلبة.

مصطلحات الدراسـة: (1) مص:

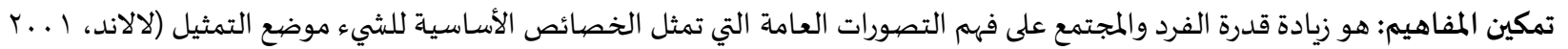
ص7 197 ) ويتم الكشف عن درجة تمكن هذه المفاهيم لدى طلبة معهد العلوم الإسلامية بمسقط عبر تطبيق اختبار تحصيلي محكم. الثورة الصيناعية الرابعة: هي العصر الصناعي الرابع التي يتميز بدمج التقنيات ببعضها، كما يتميز باختراق التكنولوجيا الناشئة في المجالات

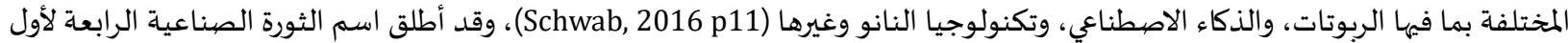
مرة في منتدى دافوس الاقتصادي بسويسرا عام 17 ـأم. 
يُعد مصطلح الثورة الصناعية الرابعة رديفا لما يطلق عليه الثورة الرقمية (Philbeck \& Davis2018 p17)، وهو مصطلح كشف عنه كلاوس شواب' (Klaus Schwab) في كتاب نشره في يناير 17 .r يحمل العنوان نفسه، "الثورة الصناعية الرابعة". ولا يمكن بأية حال من الأحوال تجاوز ثلاث الثاث

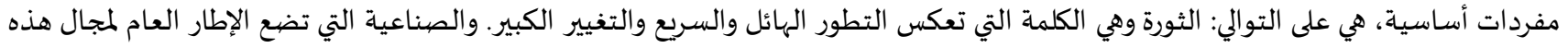

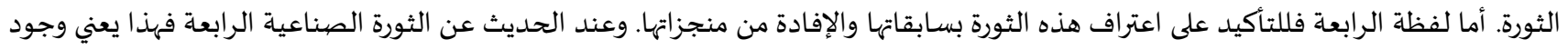

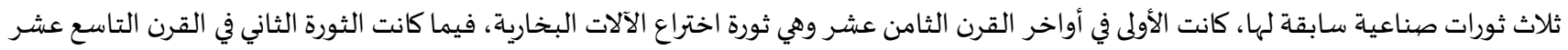

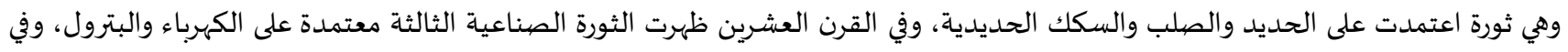
القرن الحادي والعشرين جاءت الثورة الرقمية (Philbeck\& Davis , 2018 p12-21).

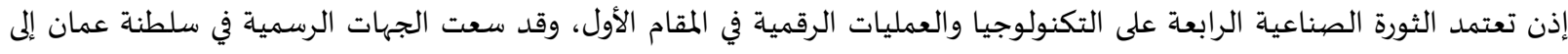

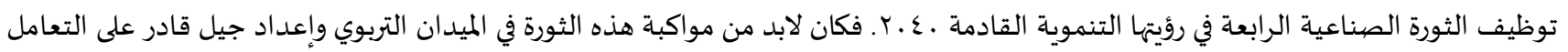

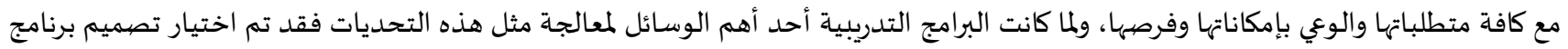

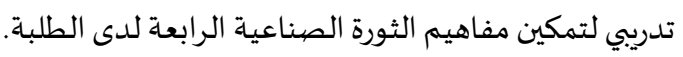

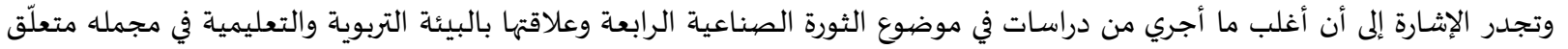

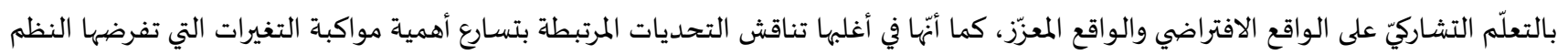

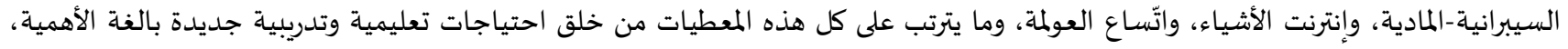

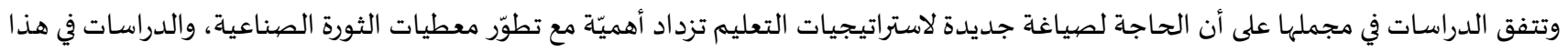

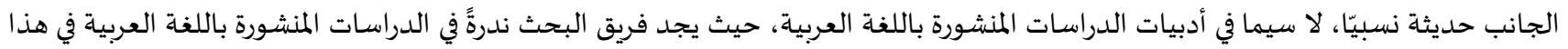

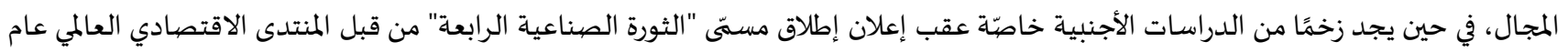

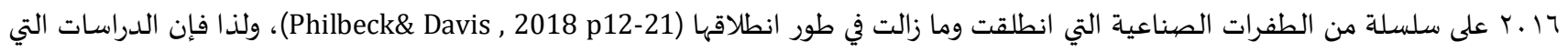

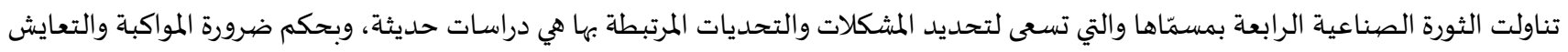

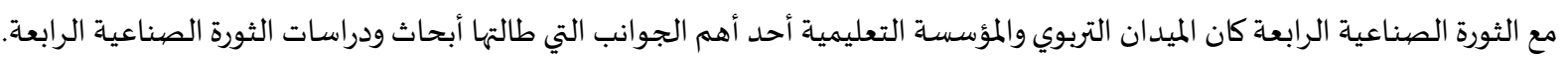

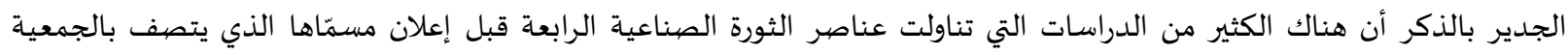

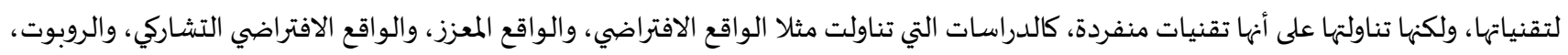

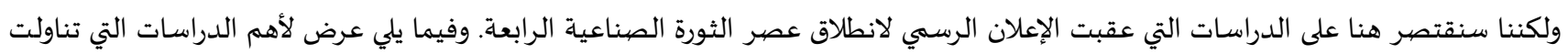

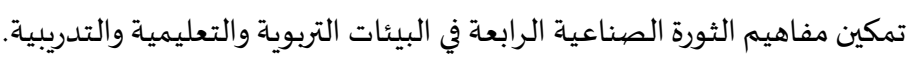

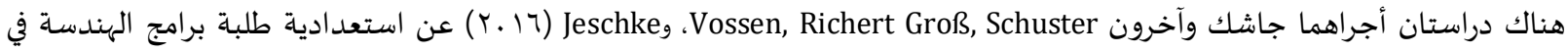

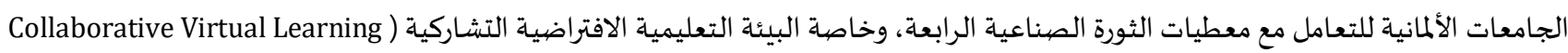

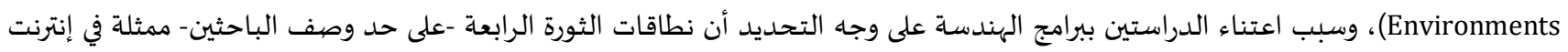

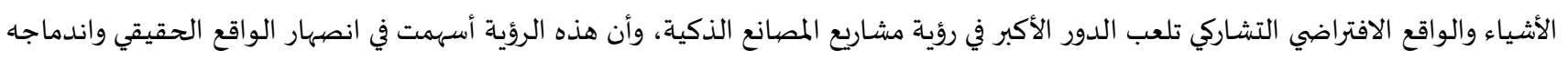

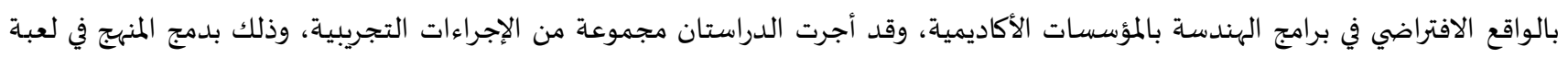
Minecrafts

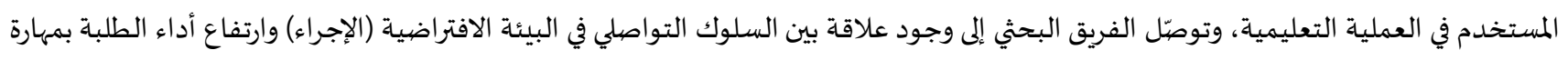

حل المشكلات تشاركيّا في البيئات الافتراضية.

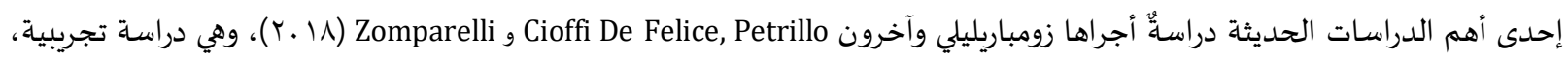

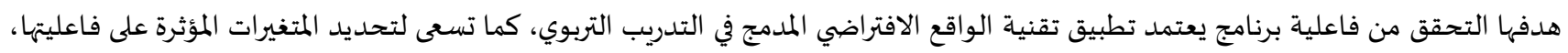

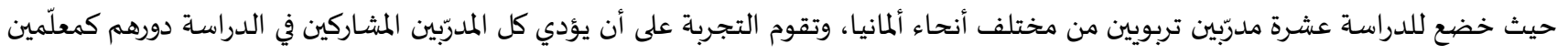

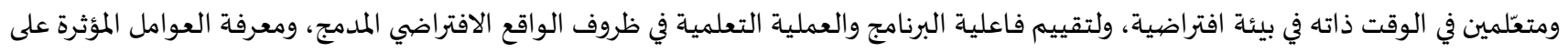

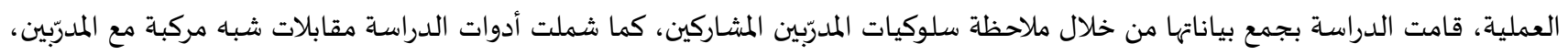

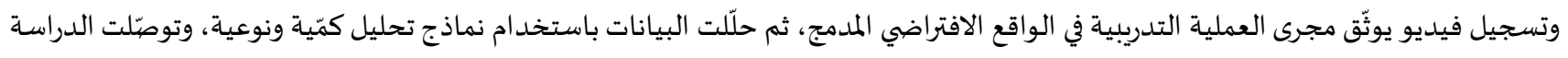

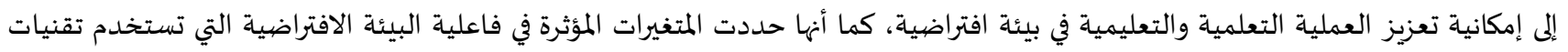

مهندس ألماني واقتصادي مؤسس منتدى الاقتصادي العالمي، ورئيسه التنفيذي. 
الواقع الافتراضي المدمج، من بين هذه المتغيرات التي حددتها الدراسة على أنها مؤثرة في أداء المدربين في بيئة تستخدم الواقع الافتراضي المدمجي: خبرة

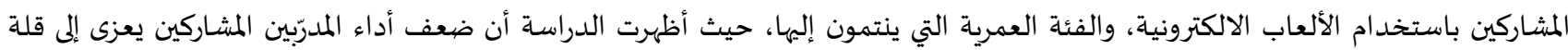

انخراطهم في بيئات الواقع الافتراضي في سنّ مبكّر.

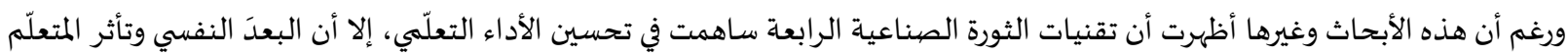

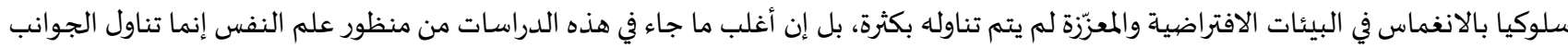

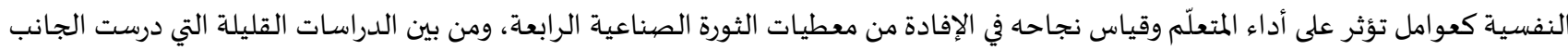

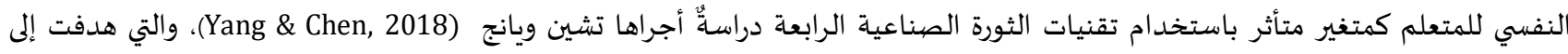

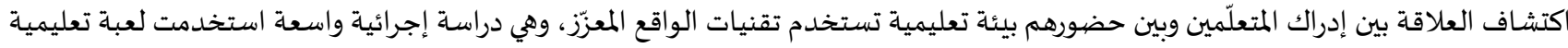

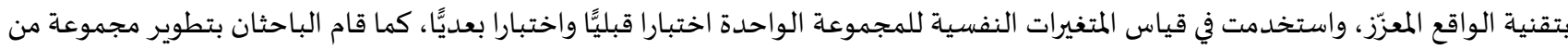

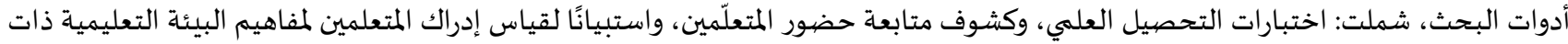

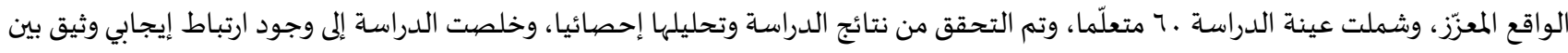

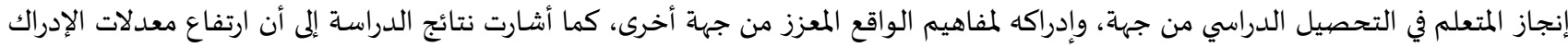

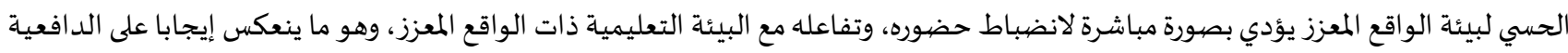

والتحصيل العلمي.

ولأن مفهوم الثورة الصناعية الرابعة مرتبط بحقبة جديدة من التصنيع الذكي الناجم عن تطور التكنولوجيا الرقمية والروبوت، تتمثل الرؤية

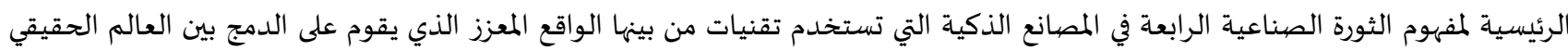

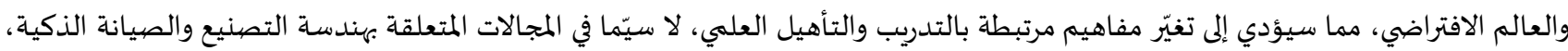

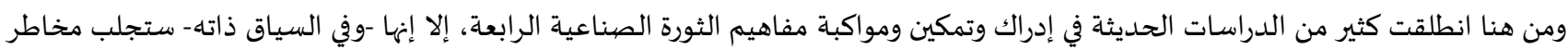

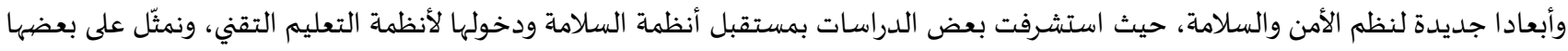

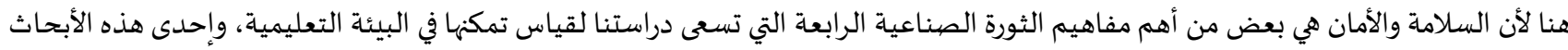

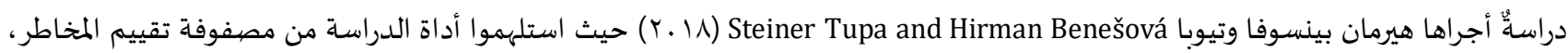

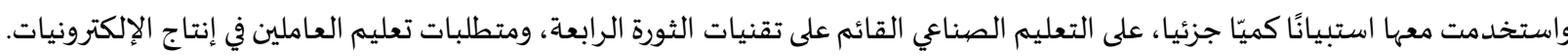

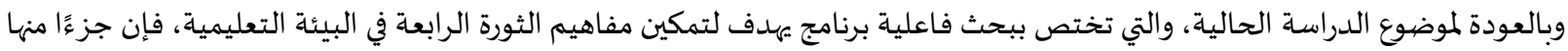

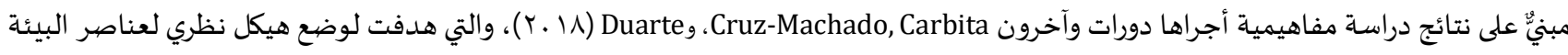

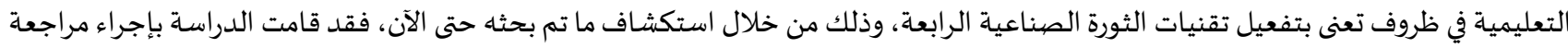

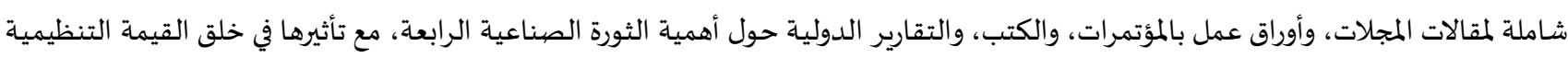
لعناصرها، وكيف يمكن لنظرية رأس المال الفكري أن تساعد في الاستفادة من فوائدها.

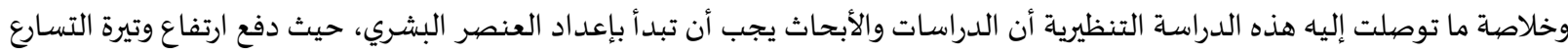

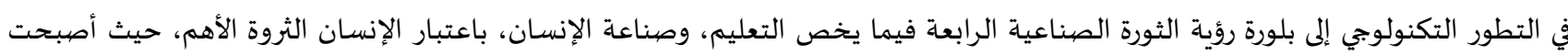

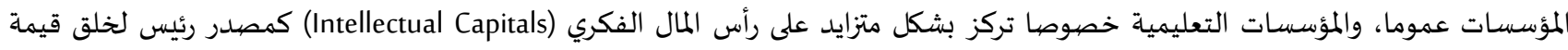

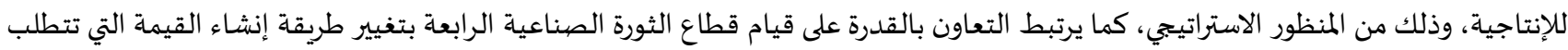

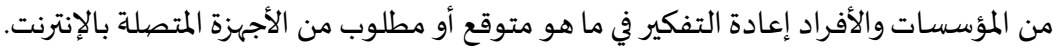

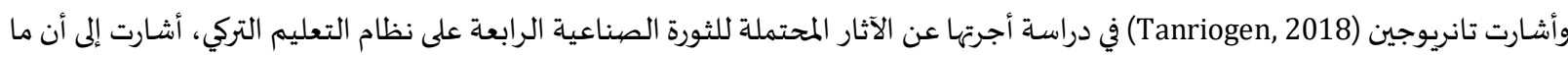

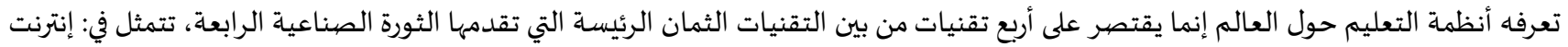

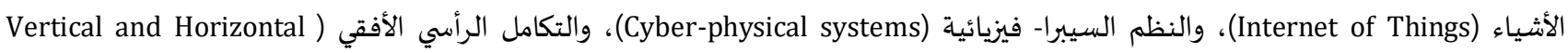

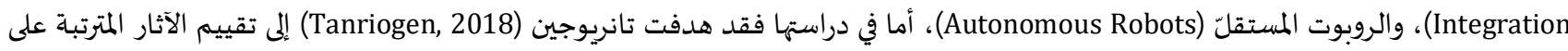

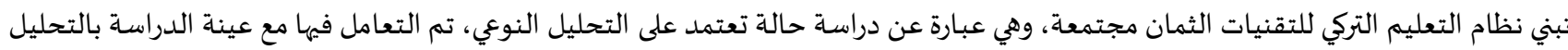

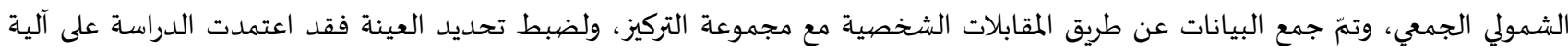

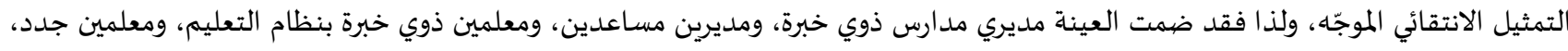

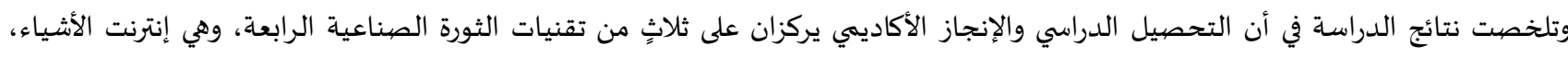

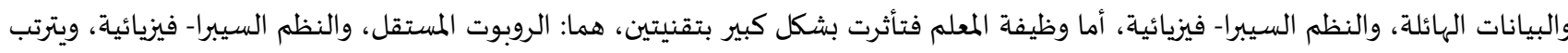

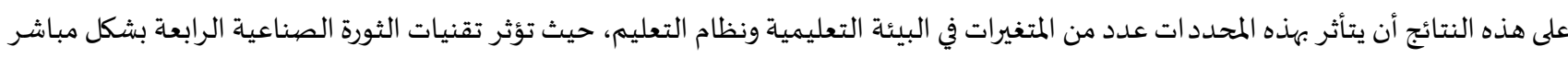


على التواصل المدرسي، والتحصيل الدراسي، والمفاهيم المدرسية، ومهامّ المعلم، والبنية الاجتماعية بشكل عامّ، وخرجت الدراسة بتوصيات أهمها أن

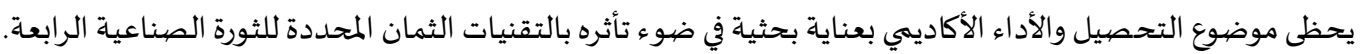
بنظرة أشمل إلى أدبيات الدراسات السابقة حول موضوع تمكّن مفاهيم الثورة الصناعية الرابعة وخصوصا في البيئات التربوية والتعليمية في

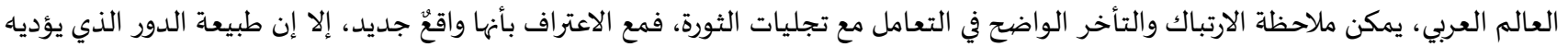

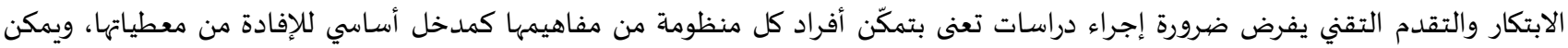

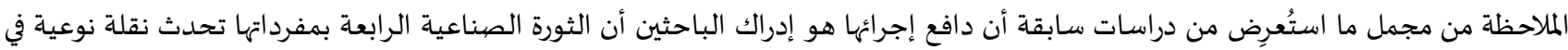

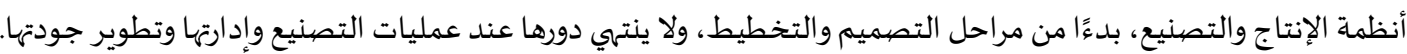

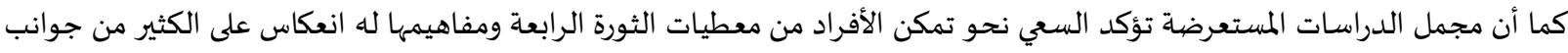

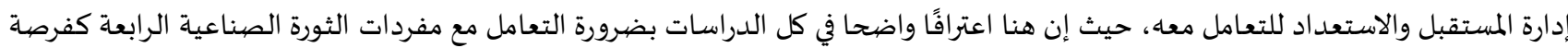

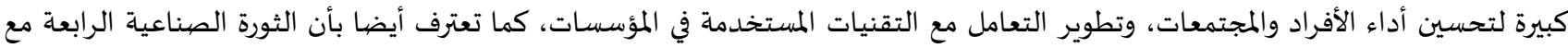

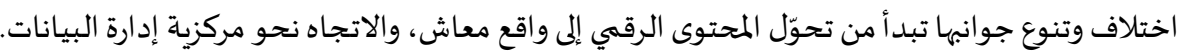
وتتفق أغلب الدراسات التي تم استعراضها في مجموعة من التوصيات، أبرزها: ضرورة تقبّل الواقع التقني الذي يفرض استخدام معطيات الثورة

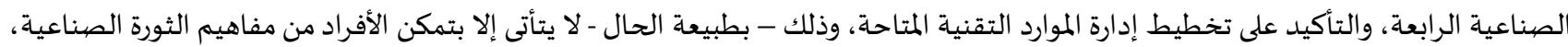
واستعدادهم لخطط التكامل والاندماج وتبادل البيانات. ومن خلال الدراسات السابقة توصلنا إلى المصطلحات الآتية:

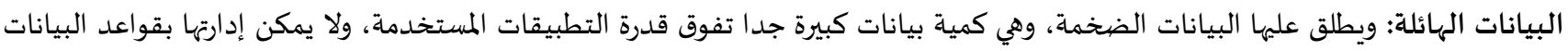

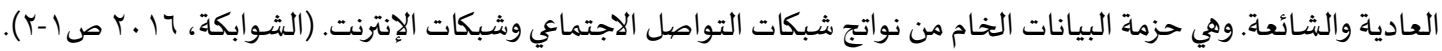
الروبوت الذاتي: هو روبوت مستقل ذاتي التحكم يستخدم لأغراض مختلفة طبية وعسكرية (Haralick \& Shapiro, 1992).

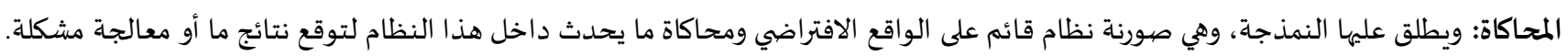
(Law \& Kelton, 2000 p1-3) إنترنت الأشياء: هو جيل جديد من الإنترنت يتيح التواصل مع الأجهزة المحيطة والتحكم بها عن طريق الإنترنت

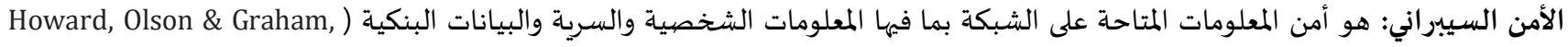
.(2016 p1

الحوسبة السحابية: هو إتاحة البيانات إلكترونيا للمستخدمين والتطبيقات والنظم الإلكترونية، تحت الطلب عبر الإنترنت ( Rittinghouse \& (Ransome, 2016 p8 \& Zukas, J.A التصنيع المضاف: ويطلق عليها الطباعة الثلاثية الأبعاد، وتتيح طباعة أجسام ثلاثية الأبعاد مما يوفر إمكانية تفريد تصنيع الأجهزة 2015 p12).(Zukas, V

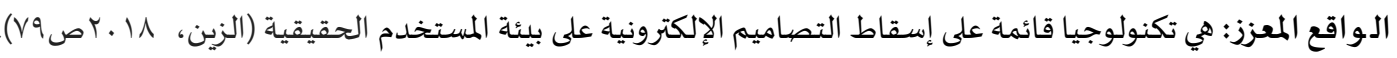
الممارسات السلوكية والرقمية: يقصد بها الفريق البحثي هو واقع تعامل الطلبة مع أجهزة التقنية الحديثة، بوصفها تجارب شخصية في توظيف التكنولوجيا في الحياة العامة. محسددات الدراسـة: الحدود المكانية: معهد العلوم الإسلامية بمسقط التابع لمركز السلطان قابوس العالي للثقافة والعلوم.

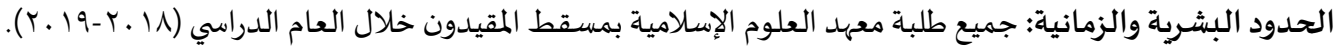
الحدود الموضيوعية: أثر برنامج تدريبي مقترح لتمكين مفاهيم الثورة الصناعية الرابعة لدى الطلبة معهد العلوم الإسلامية بمسقط.

\section{منهجية الدراسـة واجراءاتها} منهج الدراسـة:

اعتمدت هذه الدراسـة على المنهج شبه التجريبي بتصميم المجموعة الواحدة مع قياس قبلي وقياس بعدي من أجل معرفة أثر الإجراءات المطبقة في البرنامج التدريبي على تمكين مفاهيم الثورة الصناعية الرابعة لدى طلبة معهد العلوم الإسلامية بمسقط. 


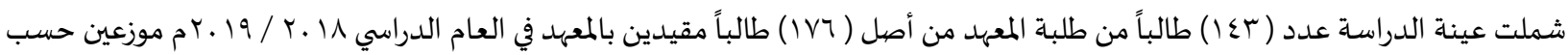
الصف الدراسي كالآتي: به طالباً في الصف العاشر، اه طالباً في الصف الحادي عشر و وب طالباً في الصف الثاني عشر، وبقية طلبة المعهد والبالغ عددهم ( سب ) طالباً تم تطبيق العينة الاستطلاعية عليهم بعد إجراء التعديلات المقترحة التي قدمها المحكمون لبعض فقرات ومحاور الاستبيان.

أداة الدراسـة:

استخدمت الدراسة الاختبار التحصيلي بوصفه أداة رئيسة تطبق على جميع أفراد العينة قبل البرنامج التدريبي وبعده، ويهدف الاختبار إلى قياس مدى تمكن الطلبة المقيدين في المعهد من مفاهيم الثورة الصناعية الرابعة ومقاصدها وأهدافها، تمهيدا لتصيميم برنامج تدريبي لتمكين هذه المفاهيم في

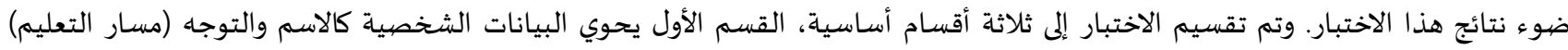

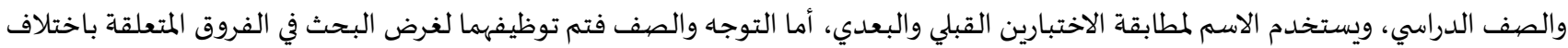

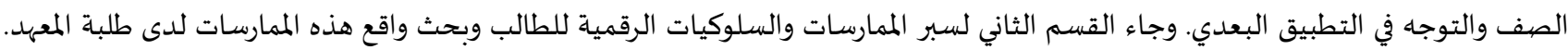

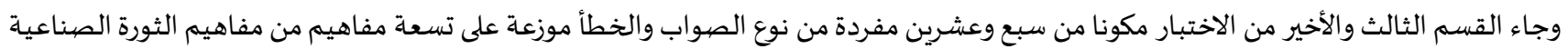

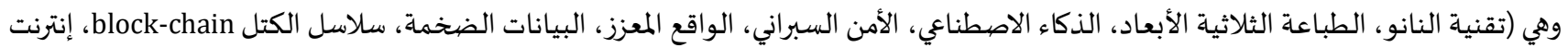

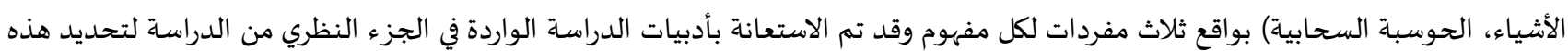
المفاهيم التسعة، وهدفت المفردات لقياس مدى تمكن هؤلاء الطلبة من هذه المفاهيم حيث تراوحت مفردات الاختبار من حيث الصياغة تهية بين سلبية

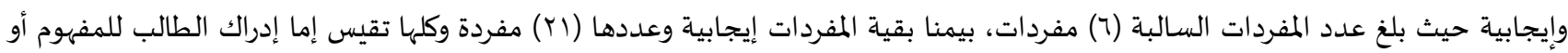
لفهما للأبعاد التطبيقية.

صدق وثبات أداة الدراسة: للتأكد من الصدق الظاهري للاختبار، تم عرضها على أربعة من المحكمين (ملحق ؟)، للحكم على درجة وضهوح المفردات وارتباطها بالموضوع المراد

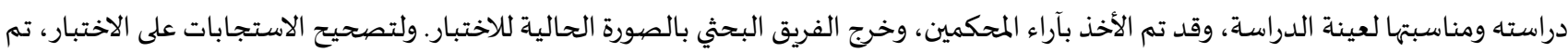
منح الدرجة (1) للمفردات المشار الهها في الاستجابة بـ ( نعم )، والدرجة (r) للمفردات المشار اليها في الاستجابة بـ ( لا )، بعد ذلك تم حساب معامل الثبات حسب طريقة الاتساق الداخلي باستخد ام معادلة (ألفا كرونباخ) حيث بلغ (VV), . ) وهو مستوى ثبات مقبول تربودياً. وبعد تطبيق الاستبيان القبلي جرى استقدام محاضرين متخصصين في مجال الثورة الصناعية الرابعة وتطبيقاتها، وعددهم (م) محاضرين وكانت

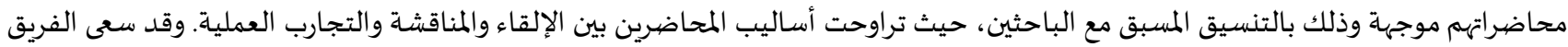

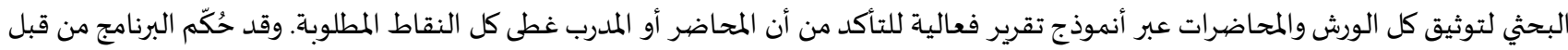

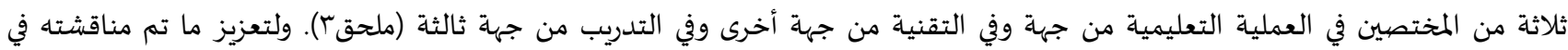
المحاضرات جرى توظيف الإذاعة الصباحية، وذلك بكتابة فقرة يوميا وبواقع ثلاث حلقات لكل مفهوم من مفاهيم الثورة الصناعية الرابعة التي تم

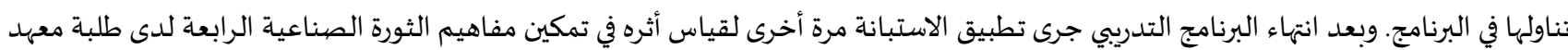

العلوم الإسلامية بمسقط.

المعالجة الإحصيائية:

بعد انتهاء البرنامج التدريبي وتطبيق الاستبيان البعدي، تم إدخال نواتج تطبيق الاستبيان في برنامج المعالجة الإحصائية (SPSS)، وقد تم احتساب التكرارات والنسب المئوية والمتوسطات الحسابية والانحرافات المعيارية، كم تم توظيف اختبار ت للعينات المستقلئلة، واختبار ت لعينتين مترابطتين، وتحليل التباين الأحادي للإجابة عن أسئلة البحث.

نتائج الدراسة ومناقشتها السؤال الأول: ما واقع الممارسات والسلوكيات الرقمية لدى طلبة معهد العلوم الإسلامية بمسقط؟ للإجابة عن السؤال الأول، تم حساب التكرارات والنسب المئوية للأجهزة الرقمية التي يستخدمها طلبة المعهد وكذلك للممارسات الرقمية التي

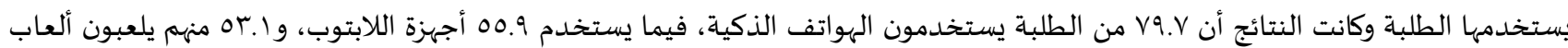
الفيديو، أما بقية الأجهزة الإلكترونية فقد تراوحت نسب استخدامها بين 7، 7r وـ.9.9. 
ويلاحظ أن أعلى ذسبة استخدام للأجهزة الرقمية من قبل طلبة المعهد كانت للهواتف الذكية وبنسبة ( V9,V \% ) وجاء بعدها مباشرة في الاستخدام الحواسيب الشخصية ( اللاب توب ) وبنسبة ( 90,\% )، بينما حصل قارئ الكتب الإلكترونية على أقل نسبة استخدام من قبل طلبة المعهد

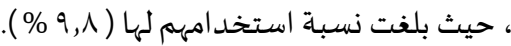

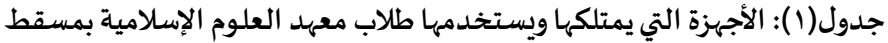

\begin{tabular}{|c|c|c|c|}
\hline النسبة المئوية & عدد الطلبة & الأجهزة الإلكترونية & م \\
\hline$r_{1},$. & $r$. & حاسوب مكتبي & 1 \\
\hline 00,9 & $\wedge$. & لابتوب & $r$ \\
\hline$r T, \varepsilon$ & rt & تاب & $r$ \\
\hline $9, \wedge$ & $1 \varepsilon$ & قارئ كتب إلكترونية & $\varepsilon$ \\
\hline$\vee १, v$ & $11 \varepsilon$ & هاتف ذكي & 。 \\
\hline$r 7,7$ & ru & MP3 & 7 \\
\hline or, 1 & $\mathrm{~V} 7$ & ألعاب فيديو & $\mathrm{r}$ \\
\hline$r u, V$ & $\mu$ & 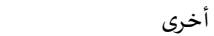 & $\Lambda$ \\
\hline
\end{tabular}

ويرى الفريق البحثي أن حصهول الهواتف الذكية على أعلى نسبة امتلاك أمر منطقي، فبالإضافة إلى أن هذه الهواتف متوفرة بكثرة، وأحيانا

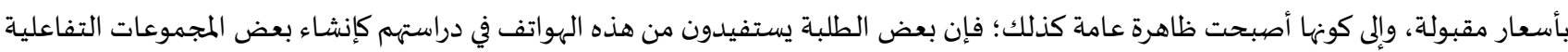
للتدارس في بعض المواد، واستخدام شبكة المعلومات العالمية فيها للبحث عما يشكل عليهم في دراستهم.

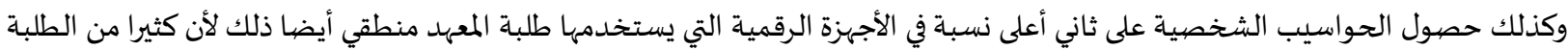
يستخدمون هذه الحواسيب في إنجاز مشاريعهم التي يكلفون بها من قبل المعلمين. بينما حصل قارئ الكتب الإلكترونية على أقل نسبة امتلاك بين طلبة المعهد وقد يعود ذلك لندرتها في الأسواق المحلية فلا يوجد وكيل معتمد للقارئات الإلكترونية في السلطنة حتى وقت تحرير هذا البحث، وإن كان يمكن

اقتنائها عبر المتاجر الإلكترونية أو الوسطاء المحليين، ويمكن أخذ جهاز الكندل أنموذجا والذي يباع -في الغالب-عبر متجر أمازون Amzon.com.

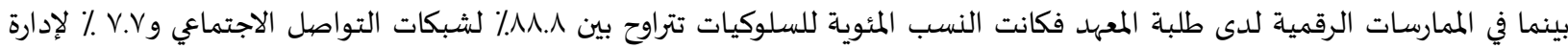

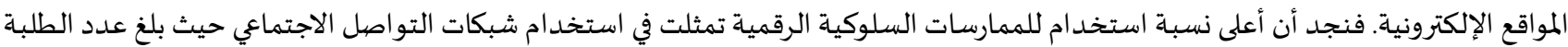

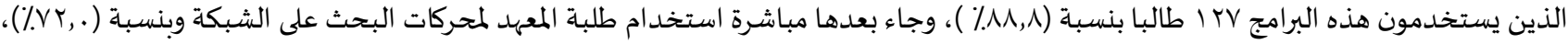
بينما جاء في المرتبة الأخيرة من بين الممارسات الأقل استخداما من قبل طلبة الماتل المعهد هي إدارة المواقع الإلكترونية حيث لم يتجاوز عدد الطلبة الذين يمارسون ذلك سوى 1 ا طالبا بنسبة (v,v).

جدول(r): الممارسات والسلوكيات الرقمية لطلاب معهد العلوم الإسلامية بمسقط المسط

\begin{tabular}{|c|c|c|c|}
\hline 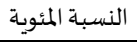 & 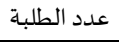 & الممارسات والسلوكيات الرقمية & r \\
\hline$r \cdot, \Lambda$ & $\varepsilon \varepsilon$ & استخدام البريد الإلكتروني للتواصل & 1 \\
\hline$v \cdot, 7$ & 1.1 & استخدام تطبيقات برامج Office & r \\
\hline 00,9 & ᄉ. & تنزيل وقراءة ملفات ومجلدات من الانترنت download & $r$ \\
\hline$V Y$, & $1 . r$ & استخدام محركات البحث على الشبكة search & $\varepsilon$ \\
\hline$v, v$ & 11 & إدارة موقع إلكتروني website & $\circ$ \\
\hline$r T, \varepsilon$ & rt & استخدام المدونات الإلكترونية & 7 \\
\hline$\varepsilon \cdot, 7$ & 01 & استخدام تطبيقات وبرامج مشاركة الملفات Drive & v \\
\hline$\Lambda, \Lambda$ & ITV & استخدام شبكات التواصل الإجتماعي & $\Lambda$ \\
\hline or, 1 & $\mathrm{~V} 7$ & ممارسات أخرى مثل ألعاب الفيديو & 9 \\
\hline
\end{tabular}

ويرجع الفريق البحثي هذه النتيجة إلى أن حصول استخدام شبكات التواصل الاجتماعي على النسبة الأعلى في امتلاك أغلب طلبة المعهد إما هواتف ذكية أو حواسيب شخصية ومن ناحية أخرى فإن هذه الشبكات وفرت فرصة لكثير من الطلبة للتعبير عن أفكارهم وآرائهم حول العديد من القضيايا المختلفة. وكذلك حصول ( البحث في الشبكات ) على المستوى الثاني من بين الممارسات السلوكية لدى طلبة المعهد ينسجم مع امتلاك أغلب الطلبة على الحواسيب الشخصية ولتوافر قاعة حاسوب في المعهد وثانية والمكتبة وأخرى في السكن الداخلي وكلها مرتبطة بشبكة الانترنت وهذا الأمر سهل على على بمات الطلبة استخدام الشبكة في البحث عن المعلومات المختلفة والمتجددة في فنون العلم. 
بينما يعزي الفريق البحثي حصول ( إدارة المواقع الإلكترونية ) على النسبة الأقل من بين الممارسات الرقمية لطلبة المعهد إلى أن هذه الممارسـة

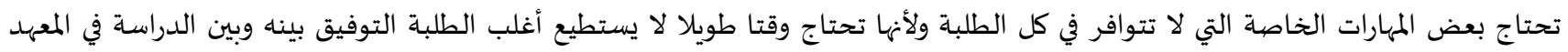
خصوصا وأن معاهد العلوم الإسلامية تتميز بزيادة عدد المواد الدراسية التي يدرسها الطالب مقارنة بأقرانهم في بعض المضاتية المؤسسات التعليمية الأخرى كمدارس وزارة التربية والتعليم.

السؤال الثاني: ما أثر البرنامج التدريبي المقترح في تمكين طلبة معهد العلوم الإسلامية بمسقط من مفاهيم الثورة الصيناعية الرابعة؟

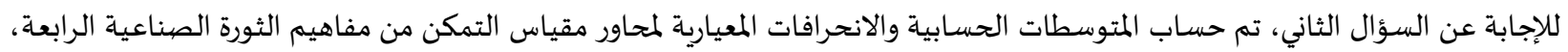

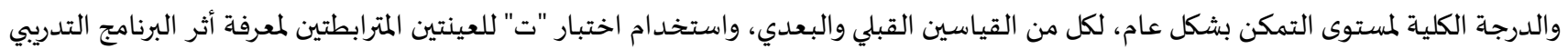
في رفع مستوى التمكن من مفاهيم الثورة الصناعية الرابعة.

جدول (r): نتائج اختبار"ت" للعينتين المتر ابطتين لمعرفة دلالة الفروق بين متوسط درجات القياسين القبلي والبعدي في مقياس التمكن من مفاهيم الثورة

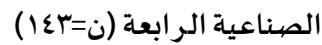

\begin{tabular}{|c|c|c|c|c|c|c|c|}
\hline \multirow[b]{2}{*}{ اتجاه الفروق } & \multirow[b]{2}{*}{ القيمة الاحتمالية } & \multirow[b]{2}{*}{ قيمة (ت) } & \multicolumn{2}{|c|}{ القياس البعدي } & \multicolumn{2}{|c|}{ القياس القبلي } & \multirow[t]{2}{*}{ المتغيرات } \\
\hline & & & $\varepsilon$ & م & $\varepsilon$ & م & \\
\hline قبلي & $\cdot, \ldots$ & $\varepsilon, . T$ & ., 19 & $1, \varepsilon \varepsilon$ & , , ז & 1,01 & الحوسبة السحابية \\
\hline بعدي & $\cdot, \ldots$ & $\varepsilon, \vee q$ &.,$r$. & 1,9 &.,$Y \Lambda$ & $1, \mathrm{VV}$ & الطباعة ثلاثية الأبعاد \\
\hline بعدي & $\cdot, \ldots$ & 7,77 &.,$r 7$ & $1, \wedge 1$ &.,$M$ & 1,70 & الواقع المعزز \\
\hline بعدي & $\cdot, \ldots$ & 7,77 & $\cdot, r 7$ & 1,11 & , Tr & 1,79 & البيانات الضخمة \\
\hline بعدي &.,$\ldots 1$ & $r, \mathrm{~V} r$ & ., r. & 1,19 & ת & 1,11 & الذكاء الاصطناعي \\
\hline بعدي & $\ldots$ & $r, r r$ &.,$Y Y$ & 1,91 &., 19 & 1,20 & النانو \\
\hline بعدي &.,$\ldots$ & 0,79 &.,$Y$ & 1,17 &.,$T$ & $1, v$. & الأمن السيبراني \\
\hline--- & ., हr. &., $\mathrm{V9}$ &.,$r T$ & $1,7 \pi$ &., $\mathrm{r \Lambda}$ & 1,71 & انترنت الاشياء \\
\hline بعدي & $\cdot, \ldots$ & $r, 9 \mathrm{~V}$ & $\cdot, \uparrow \wedge$ & $1, V \varepsilon$ & . r I & 1,71 & البلوك شين \\
\hline بعدي & $\cdot, \ldots$ & $\Lambda, r^{\prime}$ &., $1 \mathrm{r}$ & $1, v \wedge$ &., $1 \varepsilon$ & 1,71 & المقياس ككل \\
\hline
\end{tabular}

م = المتوسط الحسابي.ع = الانحراف المعياري.

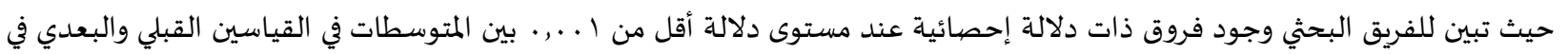
مستوى تمكن عينة الدراسـة من مفاهيم الثورة الصناعية الرابعة بشكل عام وفي جميع المحاور عدا محور "إنترنت الأشياء"، أما اتجاه باقي الفروق فجاء لصالح القياس البعدي في جميع المحاور ما عدا محور "الحوسبة السحابية"، ويعزو الفريق البحثي الاتجاه العكسي للفرق في محور "الحوسبة التهاية

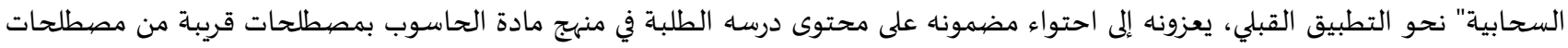

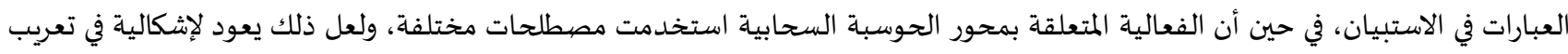

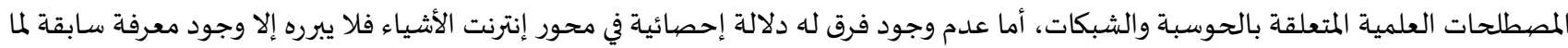

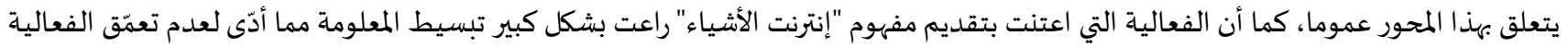
في تفاصيل قد تشكل على المرحلة التعليمية لطلبة المعهد.

وحيث إن المتوسط الحسابي للقياس البعدي عموما أعلى من المتوسط الحسابي للقياس القبلي، فإن الفروق تكون لصالح القياس البعدي، كما توضح النتائج أن أثر البرنامج المقترح للتمكن من مفاهيم الثورة الصناعية الرابعة يعتبر كبيرا، حيث أن قيمة معامل (d) بلغت (ع (1) )، ومعيار كوهين (Cohen, 1988)

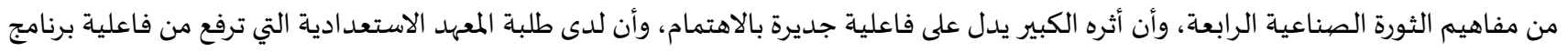
يقدماء متخصصون في مجالات الثورة الصناعية الرابعة، الأمر الذي يعزى إلى الأثر الإيجابي لبيئة المعهد التي تدعم التطور التقني، وتسعى لتوسيع آفاق لمهي الطلبة ومداركهم ليواكبوا ركب الثورة الصناعية الرابعة. وللنظر في تفاصيل أثر البرنامج في رفع مستوى تمكن الطلبة من مفاهيم الثورة الصناعية الرابعة، تم حساب المتوسطات الحسابية والانحرافات المعيارية لمحاور التمكن من مفاهيم الثورة الصناعية الرابعة، والمتوسط الكلي للتمكن بشكل عام في التطبيق البعدي، وللحكم على المتوسطات

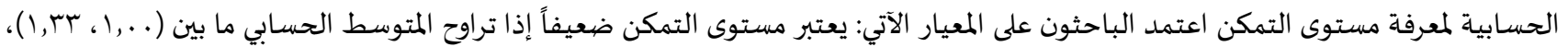

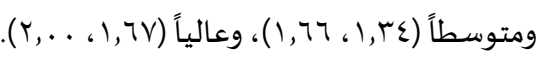

وقد اتضح للفريق البحثي أن المتوسط الكلي لمقياس التمكن من مفاهيم الثورة الصناعية الرابعة بلغ (I,VA) )، بمستوى تمكنٍ عالٍٍ، وقد تراوحت

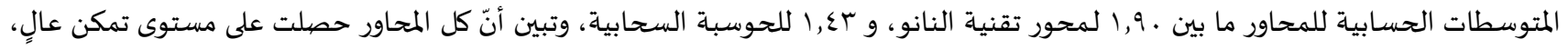


باستثناء محوري "إنترنت الأشياء"، و"الحوسبة السحابية" اللذين حصلا على مستوى تمكن متوسط، في حين حصل محور "تقنية النانو" على أعلى

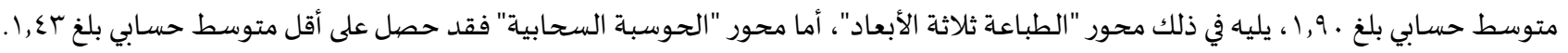
كما تم مقارنة هذه المتوسطات الفعلية بالمتوسط النظري البالغ (1,0) باستخدام اختبار "ت" للعينة الواحدة (One-Sample Test). فقد تبين

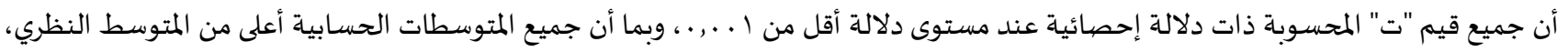
وبدلالة إحصائية في جميع محاور مقياس التمكن من مفاهيم الثورة الصناعية الرابعة وفي المتوسط الكلي، فهذه النتيجة تشير إلى أن مستوى التمكن

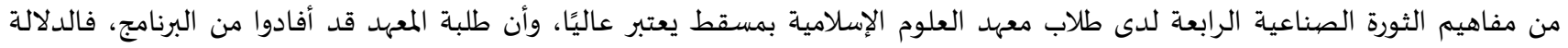

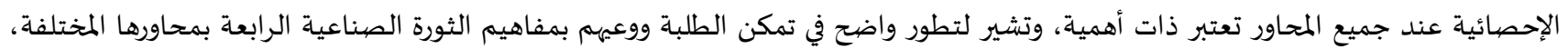
ويرى فريق البحث أن البرنامج مع كونه فاعلا حسب الدلالات الإحصائية فإن فاعليتا أفادت من البيئة العلمية للمعهد، ومن جاهزية الطلبة لمجاراة التطور العلمي في مختلف الجوانب التقنية.

السؤال الثالث هل يختلف مستوى تمكن مفاهيم الثورة الصناعية لدى طلبة المعهد باختلاف كل من الصف الدراسي ومسار التعليم؟

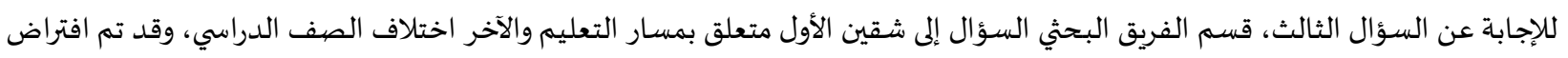

الفرضيتين الصفريتين:

• لا يختلف مستوى تمكين مفاهيم الثورة الصناعية الرابعة لدى طلبة معهد العلوم الإسلامية باختلاف الصف الدراسي. •لا توجد فروق ذات دلالة إحصائية في مستوى تمكين مفاهيم الثورة الصناعية الرابعة لدى طلبة معهد العلوم الإسلامية بمسقط بين المسار العلمي والمسار الأدبي. أولاً: متغير مسار التعليم: جدول(ع): نتائج اختبار"ت" للعينات المستقلة لمعرفة دلالة الفروق في مستوى التمكن من مفاهيم الثورة الصناعية الرابعة وفق مسار التعليم.

\begin{tabular}{|c|c|c|c|c|c|c|}
\hline \multirow[t]{2}{*}{ القيمة الاحتمالية } & \multirow[t]{2}{*}{ قيمة (ت) } & \multicolumn{2}{|c|}{ أدبي (ن=19) } & \multicolumn{2}{|c|}{ علمي (ن=|VI) } & \multirow[t]{2}{*}{ المتغيرات } \\
\hline & & $\varepsilon$ & م & $\varepsilon$ & م & \\
\hline ., \&个. & $\cdot, \lambda 1$ &.,$r Y$ & $1, r 0$ &.,$Y V$ & $1, \varepsilon$ & الحوسبة السحابية \\
\hline.,$\leqslant V V$ & $\cdot, \times 1$ &., $1 \gamma \wedge$ & 1,94 &., 19 & 1,19 & طباعة الأبعاد الثلاثية \\
\hline.,$\varepsilon \vee$. &.,$V T$ &.,$Y \wedge$ & $1, \lambda r$ & . & $\mathrm{I}, \mathrm{AV}$ & الواقع المعزز \\
\hline.,$r q$. & $1, T V$ & .r.r. & $1, \mathrm{VA}$ & . & 1,17 & البيانات الضخمة \\
\hline . TrA & 1,19 &.,$r$ & $1, \wedge \varepsilon$ &., 10 & 1,9 & الذكاء الاصطناعي \\
\hline., 011 &., $0 \varepsilon$ &.,$T V$ & 1,19 &., 197 & $1,9 r$ & النانو \\
\hline., $9 \mathrm{rV}$ &., .1 &.,$Y T$ & 1,17 &., $1 T$ & 1,17 & الأمن السيبراني \\
\hline .,, 191 & . TV &.,$r$ & 1,70 & . & $1, \pi r$ & انترنت الأشياء \\
\hline .,940 &., .1 &.,$Y \wedge$ & $1, v \varepsilon$ &.,$Y \Lambda$ & $1, V \varepsilon$ & البلوك شين \\
\hline . rVq &., 19 &., $1 \varepsilon$ & 1,177 &., 1 . & $1, \sqrt{ } 9$ & المقياس ككل \\
\hline
\end{tabular}

م:المتوسط الحسابي، ع: الانحراف المعياري

للإجابة عن الشق المتعلق بمتغير مسار التعليم، فقد تم احتساب المتوسطات الحسابية والانحرافات المعيارية لمحاور مقياس التمكن من مفاهيم

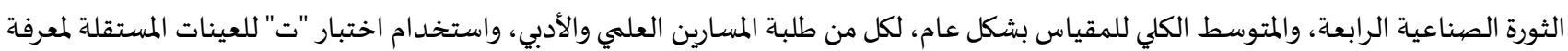
أثر متغير مسار التعليم في مستوى التمكن من مفاهيم الثورة الصناعية الرابعة، وقد اتضح من خلال النتائج عدم وجود فروق ذات دلالة إحصائية

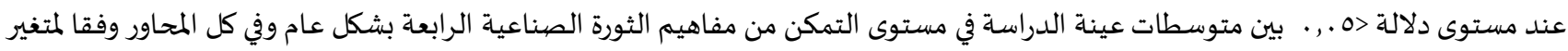
مسار التعليم. وهذا يعني اتفاق نتائج البحث مع الفرضية الصفرية التي تنص (لا توجد فروق ذات دلالة إحصائية في مستوى تمكين مفاهيم الثورة

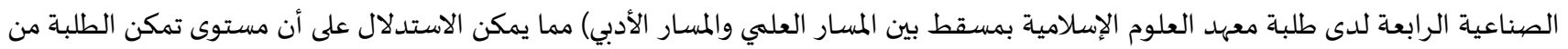
مفاهيم الثورة الصناعية الرابعة متساوي بغض النظر عن اختلاف المسار أو توجه الطالب. ثانياً: متغير الصفف الدراسي: ولإجابة عن الشق الثاني من السؤال والمتعلق بالصف الدراسي، فقد استخدم الفريق البحثي تحليل التباين الأحادي لمعرفة طبيعة الفروق بين المتوسطات الحسابية، وبعد قراءة خلاصة نتائج تحليل التباين الأحادي تبين أن قيمة "ف" المحسوبة دالة إحصائيا عند مستوى دلالة ا ... في مقياس

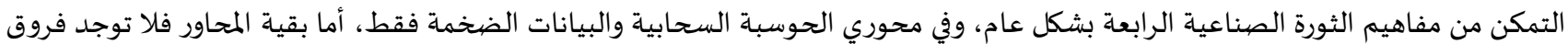

ذات دلالة إحصائية فيها. 
جدول (0): ا لمتوسط الحسابي والانحراف المعياري لمقياس التمكن من مفاهيم الثورة الصناعية الرابعة ومحوري الحوسبة السحابية والبيانات الضخمة، في مستويات الصف الدراسي منيم الصورة الصيناعي

\begin{tabular}{|c|c|c|c|c|c|c|c|}
\hline \multicolumn{2}{|c|}{ البيانات الضخمة } & \multicolumn{2}{|c|}{ الحوسبة السحابية } & \multicolumn{2}{|c|}{ المتوسط الكلي } & \multirow[t]{2}{*}{ العدد } & \multirow[t]{2}{*}{ الصف } \\
\hline$\varepsilon$ & م & $\varepsilon$ & م & $\varepsilon$ & م & & \\
\hline.,$Y 7$ & $1, V \varepsilon$ &., 49 & $1,0$. &., $1 T$ & $1, V V$ & or & العاشر \\
\hline.,$r 9$ & l,A. &., 19 & $1, r T$ &., 14 & 1,20 & 01 & الحادي عشر \\
\hline., $\mathrm{IV}$ & 1,9 . & . , Y & $1,0$. &., .9 & $1, \lambda r$ & rq & الثاني عشر \\
\hline
\end{tabular}

م: المتوسط لحسابي، ع: الانحراف المعياري

كما استُخدم اختبار شـافية للمقارنات البعدية المتعددة، وأظهرت نتائج المتوسط الكلي للمقياس وجود فروق ذات دلالة إحصائية بين الصف

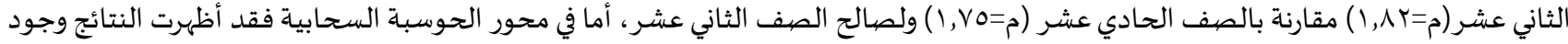

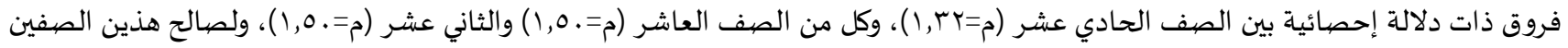

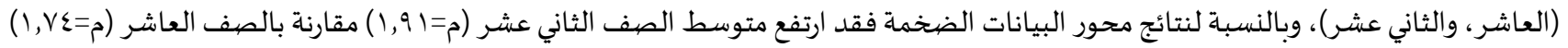

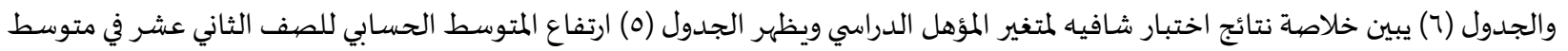

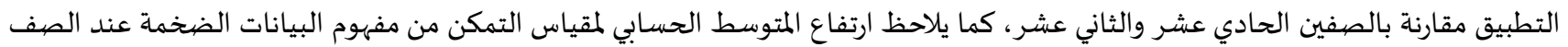
الثاني عشر متقدما على الصفين الحادي عشر والعاشر، فيما يتساوى الصفين العاشر والثاني عشر تقريبا في التمكن من مفهوم الحوسبة السحابية

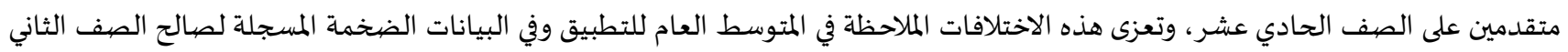
عشر لأسباب تتعلق بتقدمهم في التكوين على أقرانهم ونضجههم المعرفي، فيما يمكن تفسير تفوق العاشر على الحادي عشر في الحوسبة السحابية وذلك

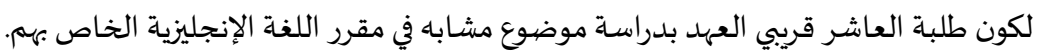

جدول (7): نتائج اختبارشافيه لمتغير متغير الصف الدراسي

\begin{tabular}{|c|c|c|c|c|c|}
\hline اتجاه الفروق & القيمة الاحتمالية & فرق المتوسطات & \multicolumn{2}{|c|}{ الصف الدراسي } & المتغير \\
\hline العاشر & $\ldots, \ldots$ & $*_{.}^{*}, 11$ & الحادي عشر & العاشر & الحوسبة السحابية \\
\hline-- & $1, \ldots$ &.,. & الثاني عشر & العاشر & \\
\hline الثاني عشر &., $.1 \varepsilon$ &., $1 \mathrm{~V}-$ & الثاني عشر & الحادي عشر & \\
\hline-- &., 0.7 &., $.7-$ & الحادي عشر & العاشر & البيانات الضخمة \\
\hline الثاني عشر & $\ldots$ & . IV- & الثاني عشر & العاشر & \\
\hline-- & . ITV & ., $11-$ & الثاني عشر & الحادي عشر & \\
\hline-- & . &.,.$r$ & الحادي عشر & العاشر & المتوسط ككل \\
\hline-- & ., $1 \varepsilon$. &.,. . $\vee \wedge \vee-$ & الثاني عشر & العاشر & \\
\hline الثاني عشر & $., . r)$ & - . . $7119-$ & الثاني عشر & الحادي عشر & \\
\hline
\end{tabular}

التوصيات والمقترحات:

وبعد النتائج التي توصلت إليها الدراسة يوصي الفريق البحثي بـ

تضمين محتوى البرنامج التدريبي داخل مقرر الحاسوب لطلبة الصف الحادي عشر.

تضمين البرنامج لبعض الجوانب التطبيقية لتوسيع الفرق بين نتيجتي تطبيق الاستبيان القبلية والبعدية.

تصميم وتنفييذ مقاطع مرئية، أو تطبيقات تفاعلية تعرض مفاهيم الثورة الصناعية الرابعة.

إجراء دراسة معمقة حول اختيارات الطلبة الدراسية المستقبلية ومدى مطابقتها للخطط التنموية في البلاد.

تقديم بحث اجرائي يبحث السبل المثلى لتمكين الوعي بالمفاهيم عموما، وإمكان تطبيقها في البيئة المعهدية.

إجراء دراسة علمية تبحث إمكان توظيف النظريات اللسانية الحديثة في تمكين مفاهيم الثورة الصناعية بصورة فاعلة وعملية تستثمر الوقت

والجهد. 


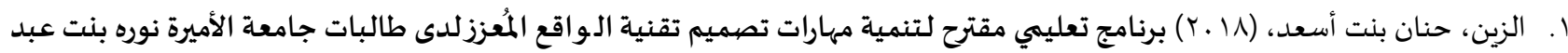

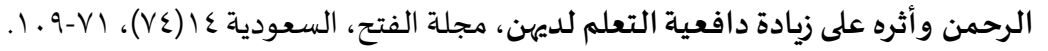

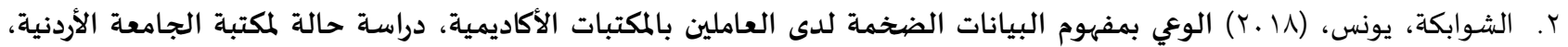

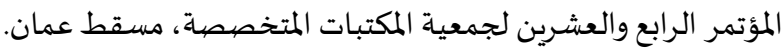

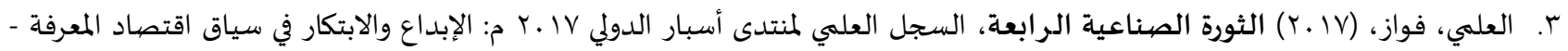

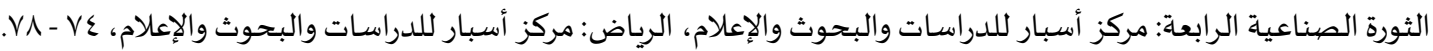

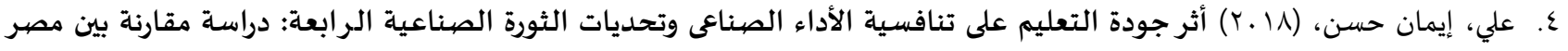

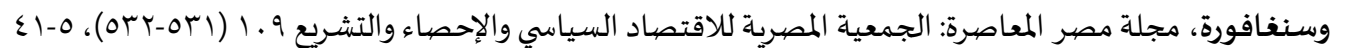

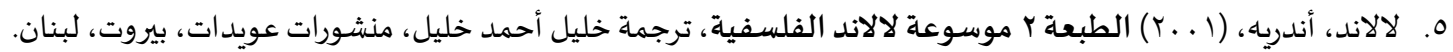

ثانياً: المراجع الأجنبية:

[1] Benešová. A., Hirman. M., Steiner. F., \& Tupa. J., Analysis of Education Requirements for Electronics Manufacturing within Concept Industry 4.0, In 2018 41st International Spring Seminar on Electronics Technology (ISSE) (2018), (pp. 1-5). IEEE.

[2] Chen. Y. H., \& Wang. C. H., Learner presence, perception, and learning achievements in augmented-realitymediated learning environments, Interactive Learning Environments, 26(5) (2018), 695-708, https://doi.org/10.1080/10494820.2017.1399148

[3] Graham. J., Olson. R., \& Howard. R., Cyber security essentials, Auerbach Publications, (2016).

[4] Haralick. R. M., \& Shapiro. L. G., Computer and robot vision (Vol. 1, pp. 28-48). Reading: Addison-wesley, (1992).

[5] Law. A. M., \& Kelton. W. D., Simulation modeling and analysis (Vol. 3). New York: McGraw-Hill, (2000).

[6] Petrillo. A., De Felice. F., Cioffi. R., \& Zomparelli. F., Fourth industrial revolution: Current practices, challenges, and opportunities, In Digital Transformation in Smart Manufacturing. InTech, (2018), https://doi.org/10.5772/intechopen.72304

[7] Philbeck. T., \& Davis. N., The Fourth Industrial Revolution: Shaping a New Era., Journal of International Affairs, 72(1) (2018), 17-22.

[8] Rittinghouse. J. W., \& Ransome. J. F., Cloud computing: implementation, management, and security, CRC press, (2016).

[9] Schuster. K., Groß. K., Vossen. R., Richert. A., \& Jeschke. S., Preparing for industry 4.0-collaborative virtual learning environments in engineering education, In Engineering Education 4.0 (pp. 477-487). Springer, Cham, (2016).

[10] Schwab. K., The Fourth Industrial Revolution, World Economic Forum, Geneva Switzerland, (2016).

[11] Tanriogen. Z. M., African University Students' Intercultural Experiences with Impeding Factors: Case from Northern Cyprus, Eurasian Journal of Educational Research (EJER), 18(77) (2018),1-24, https://doi.org/10.14689/ejer.2018.77.5

[12] Zukas. V., \& Zukas. J. A., An Introduction to 3D Printing. Sarasota, FL: First Edition Design Publishing, (2015) Retrieved from: http://search. ebscohost.com/login.aspx?direct=true \&db=nlebk\&AN=1003128\&lang=ar\&site=edslive \&scope $=$ site 


$$
\text { المجلة الدولية للدراسـات التربوية والنفسية }
$$

International Journal of Educational \& Psychological Studies (EPS)

Journal Homepage: https://www.refaad.com/views/EPSR/Home.aspx

www.refaad.com

ISSN: 2520-4149 (Online) 2520-4130 (Print)

\title{
The Effect of a Training Program on Empowering the Concepts of Industry 4.0 among Students of Muscat Islamic Institute
}

\author{
Luqman bin Khalfan al-Mayahi ${ }^{1}$, Nasr bin Nasser al-Jabri ${ }^{2}$, Abdullah bin Ali al-Jahuri ${ }^{3}$, Hussein \\ bin Ali al-Kharusi 4 \\ ${ }^{1}$ Master of Teaching Methods, Assistant Director, Institute of Islamic Sciences, Muscat, Sultanate of Oman \\ ${ }^{2}$ Master of Teaching Methods, English teacher, Institute of Islamic Sciences, Muscat, Sultanate of Oman \\ ${ }^{3}$ Master of Arabic Language and Literature, Arabic teacher, Institute of Islamic Sciences, Muscat, Sultanate of Oman \\ ${ }^{4}$ Associate Professor, Department of Psychology, Sultan Qaboos University, Sultanate of Oman \\ 1 E-mail: luqman1233@gmail.com
}

DOI : https://doi.org/DOI:10.31559/EPS2020.7.3.5

\begin{abstract}
The present study aimed to identify the effect of a training program on empowering the concepts of Industry 4.0 among the students of Muscat Islamic Institute, and whether there were statistically significant differences attributed to the variables of (1) the grade and (2) the educational orientation (Natural Sciences vs. Human Sciences). To achieve the objectives of the study, nine basic Industry 4.0 concepts were addressed in a training program designed by the researchers to approach and serve the empowerment of the nine concepts. The study population consisted of 143 of the institute students (10,11 and 12-graders). The study adopted the semiexperimental approach with a pre-test post-test model using a questionnaire. The results of the study showed a statistically significant difference in the arithmetic mean of the results of the two applications, in favor of the posttest questionnaire. The study also showed a statistically significant difference in the effect of the grade variable. The study concludes with a set of recommendations and future research suggestions.
\end{abstract}

Keywords: Industry 4.0; Empowering Concepts; Training Program.

\section{References:}

[1] 'ly. Eyman Hsn, Athr Jwdh Alt'elym 'Ela Tnafsyh Alada' Alsna'a Wthdyat Althwrh Alsna'yh Alrab'h: Drash Mqarnh Byn Msr Wsnghafwrh, Mjlt Msr Alm'asrh: Aljm'yh Almsryh Llaqtsad Alsyasy Walehsa' Waltshry', 109 (531-532)5-14, (2018).

[2] Al'lmy. Fwaz, Althwrh Alsna'yh Alrab'h, Alsjl Al'lmy Lmntda Asbar Aldwly 2017 M: Alebda' Walabtkar Fy Syaq Aqtsad Alm'rfh - Althwrh Alsna'yh Alrab'h: Mrkz Asbar Lldrasat Walbhwth Wale'lam, Alryad: Mrkz Asbar Lldrasat Walbhwth Wale'lam, (2017), 74- 78.

[3] Benešová. A., Hirman. M., Steiner. F., \& Tupa. J., Analysis of Education Requirements for Electronics Manufacturing within Concept Industry 4.0, In 2018 41st International Spring Seminar on Electronics Technology (ISSE) (2018), (pp. 1-5). IEEE.

[4] Chen. Y. H., \& Wang. C. H., Learner presence, perception, and learning achievements in augmented-realitymediated learning environments, Interactive Learning Environments, 26(5) (2018), 695-708, https://doi.org/10.1080/10494820.2017.1399148

[5] Graham. J., Olson. R., \& Howard. R., Cyber security essentials, Auerbach Publications, (2016).

[6] Haralick. R. M., \& Shapiro. L. G., Computer and robot vision (Vol. 1, pp. 28-48). Reading: Addison-wesley, (1992).

[7] Laland. Andryh, Altb'h 2 Mwsw'h Laland Alflsfyh, Trjmh Khlyl Ahmd Khlyl, Mnshwrat 'wydat, Byrwt, Lbnan, (2001).

[8] Law. A. M., \& Kelton. W. D., Simulation modeling and analysis (Vol. 3). New York: McGraw-Hill, (2000). 
[9] Petrillo. A., De Felice. F., Cioffi. R., \& Zomparelli. F., Fourth industrial revolution: Current practices, challenges, and opportunities, In Digital Transformation in Smart Manufacturing. InTech, (2018), https://doi.org/10.5772/intechopen.72304

[10] Philbeck. T., \& Davis. N., The Fourth Industrial Revolution: Shaping a New Era., Journal of International Affairs, 72(1) (2018), 17-22.

[11] Rittinghouse. J. W., \& Ransome. J. F., Cloud computing: implementation, management, and security, CRC press, (2016).

[12] Schuster. K., Groß. K., Vossen. R., Richert. A., \& Jeschke. S., Preparing for industry 4.0-collaborative virtual learning environments in engineering education, In Engineering Education 4.0 (pp. 477-487). Springer, Cham, (2016).

[13] Schwab. K., The Fourth Industrial Revolution, World Economic Forum, Geneva Switzerland, (2016).

[14] Alshwabkh. Ywns, Alw'y Bmfhwm Albyanat Aldkhmh Lda Al'amlyn Balmktbat Alakadymyh, Drast Halh Lmktbh Aljam'h Alardnyh, Alm'tmr Alrab' Wal'shryn Ljm'yt Almktbat Almtkhssh, Msqt 'man, (2018).

[15] Tanriogen. Z. M., African University Students' Intercultural Experiences with Impeding Factors: Case from Northern Cyprus, Eurasian Journal of Educational Research (EJER), 18(77) (2018),1-24, https://doi.org/10.14689/ejer.2018.77.5

[16] Zukas. V., \& Zukas. J. A., An Introduction to 3D Printing. Sarasota, FL: First Edition Design Publishing, (2015) Retrieved from: http://search. ebscohost.com/login.aspx?direct=true\&db=nlebk\&AN=1003128\&lang=ar\&site=edslive\&scope $=$ site

[17] Alzyn. Hnan Bnt As'd, Brnamj T'lymy Mqtrh Ltnmyt Mharat Tsmym Tqnyt Alwaq' Almu'zz Lda Talbat Jam't Alamyrh Nwrh Bnt 'bd Alrhmn Wathrh 'la Zyadht Daf'yt Alt'lm Ldyhn, Mjlt Alfth, Als'wdyh 14(74) (2018), 71-109. 


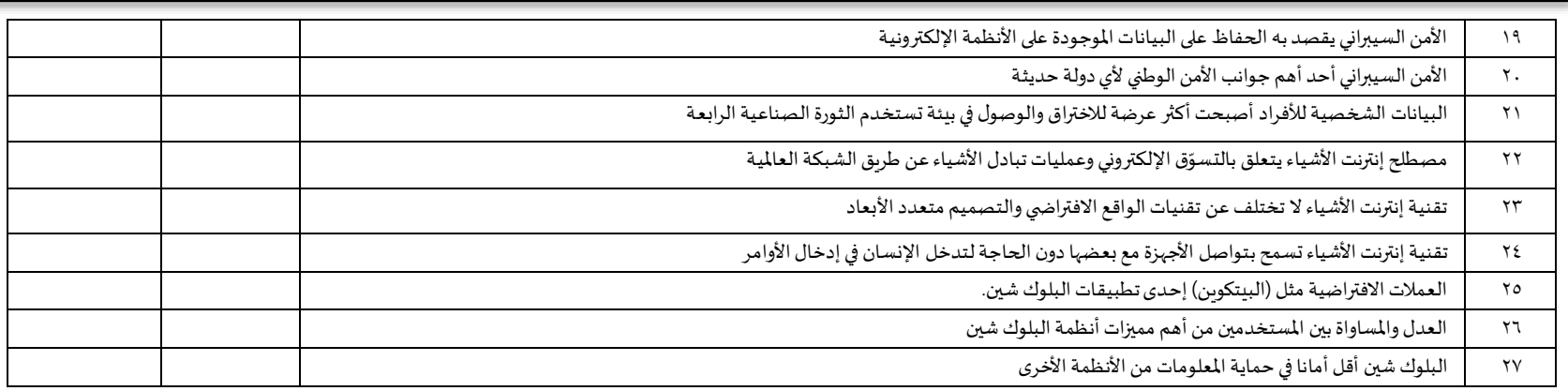

انتهى الاختبارشكرا لكم على التعاون 